\title{
THREE-DIMENSIONAL MODEL OF METASTATIC TUMOR ANGIOGENESIS IN RESPONSE TO ANTI-ANGIOGENIC FACTOR ANGIOSTATIN
}

\author{
GAIPING ZHAO*, ${ }^{*}$, ERYUN CHEN ${ }^{\dagger}$, XIAOLI YUं, HAIPO CUI*, \\ JIE LV* and JIE WU\$ \\ ${ }^{*}$ School of Medical Instrument and Food Engineering \\ University of Shanghai for Science and Technology \\ Shanghai 200093, P. R. China \\ †School of Energy and Power Engineering \\ University of Shanghai for Science and Technology \\ Shanghai 200093 P. R. China \\ \$Fudan University Shanghai Cancer Center \\ Shanghai 200032, P. R. China \\ $\$$ School of Naval Architecture \\ Ocean and Civil Engineering \\ Shanghai Jiao Tong University \\ Shanghai 200240, P. R. China \\ ףzgp_06@126.com
}

Received 15 March 2016

Revised 10 March 2017

Accepted 11 May 2017

Published 22 June 2017

\begin{abstract}
Surgeons observed that primary tumors are capable of suppressing the growth of their metastases by generating anti-angiogenic factor angiostatin. A three-dimensional (3D) mathematical model of development of the metastatic tumor vasculature is presented to simulate the morphology and construction of 3D microvascular networks under the inhibitory effect of antiangiogenic factor angiostatin excreted by the primary tumor. The simulation results demonstrate that metastatic tumor microvascular density (MVD) decreases by about $60 \%, 58 \%$ and $52 \%$, respectively, at $t=3,7$ and 14 days under the effect of anti-angiogenic factor angiostatin. The abnormal geometric and morphological features of $3 \mathrm{D}$ microvasculature networks inside and outside the metastatic tumor improve in the presence of angiostatin. The present model may allow to simulate experimental tests and may provide theoretical models for clinical research of anti-angiogenic therapy strategies.
\end{abstract}

Keywords: Metastatic tumor; angiogenesis; effect of angiostatin; 3D mathematical model.

I Corresponding author.

This is an Open Access article published by World Scientific Publishing Company. It is distributed under the terms of the Creative Commons Attribution 4.0 (CC-BY) License. Further distribution of this work is permitted, provided the original work is properly cited. 


\section{Introduction}

The growth of a tumor depends on both the tumor origin and the surrounding environment. Angiogenesis has a crucial correlation with tumor growth, invasion and metastasis. ${ }^{1}$ Angiogenesis accelerates the development of tumor and provides the oxygen and nutritions required for continued tumor growth. In addition, tumor angiogenesis also provides the initial route for invading cancer cells to escape from a primary tumor and enter in the circulation. Cancer cells migrate into the bloodstream and the surrounding tissues via the microcirculation then continue to grow giving rise to metastases ${ }^{2}$ far more difficult to treat clinically. Metastasis is the primary cause of death for most cancer patients ${ }^{3}$ and is also the important biological characteristic of malignant tumors.

The phenomenon of inhibition of metastatic tumor growth by primary tumor was observed in both clinical and laboratory studies. For example, the experimental studies carried out by O'Reilly et $a l^{4}{ }^{4}$ on animals demonstrated that some primary tumors are capable of suppressing the growth of their metastases by generating anti-angiogenic factor angiostatin. Angiostatin is a $38-\mathrm{kD}$ internal peptide of plasminogen, which is a potent inhibitor of angiogenesis in vivo, and selectively inhibits endothelial-cell (EC) proliferation and migration in vitro. ${ }^{5}$ The cells of the primary tumor secrete angiostatin which enters the blood circulation via the vascular network. The angiostatin, by virtue of its longer half-life in the circulation, ${ }^{6}$ is then transported through the bloodstream and eventually reaches the parent vessel where an angiogenic response is being elicited by the metastatic tumor. Metastatic tumor angiogenesis is a process whereby capillary sprouts are formed in response to externally supplied chemical stimuli, which is characterized by sprouting of ECs from pre-existing vasculature, tube and loop formation and the establishment of functional flow. Metastatic tumor angiogenesis is regulated by the productions of angiogenic stimulators and angiogenic inhibitors. If the level of angiogenic inhibitor such as angiostatin exceeds the level of angiogenic stimulators then metastatic tumor angiogenesis can be inhibited or prevented. Although some theoretical and experimental researches ${ }^{4,5,7}$ have been devoted to this subject, the mechanisms of anti-angiogenic activity of angiostatin are not yet fully understood.

Mathematical models that describe the tumor-induced angiogenesis ${ }^{8-16}$ and anti-angiogenic therapy ${ }^{7,17-22}$ have been developed to better understand pathophysiologic process of tumor growth and discover possible cancer therapy strategy. Maggelakis ${ }^{18}$ presented a mathematical model to examine the effects of tumor angiogenesis factor (TAF) and tumor inhibitor factors (TIFs) on tumor angiogenesis and predict the onset of vascularization. Anderson and Chaplain ${ }^{7}$ presented a one-dimensional mathematical model describing the angiogenic response of ECs to a secondary tumor and gave a possible explanation for this suppression of secondary tumors by the primary tumor. However, this model cannot present microvascular morphology and architecture in the metastatic tumor because of 
one-dimensional space restriction. Orme and Chaplain ${ }^{19}$ presented a two-dimensional (2D) mathematical model of the development of tumor vasculature to simulate anti-angiogenesis strategies and examine the roles of haptotaxis and chemotaxis in the growth of the neovasculature. Tee and DiStefano ${ }^{17}$ developed a $2 \mathrm{D}$ mathematical model of extratumoral angiogenesis in cancer in response to specific anti-angiogenic drug treatment and discussed the drug delivery and clearance rate dependencies. In addition, Alarcón et al. ${ }^{21}$ developed a multiscale model of vascular tumor growth and investigated vessel dematuration and the effects on tumor growth of combinations of anti-angiogenic and standard cytotoxic drugs. Ledzewicz and Schättler ${ }^{22}$ considered a mathematical model for tumor antiangiogenesis as an optimal control problem and analyzed the problem of how to schedule a given amount of angiogenic inhibitors to achieve a maximum reduction in the primary cancer volume. However, 3D modeling of anti-angiogenesis treatment for metastatic tumor is still not very common in literature although it would allow to better target angiogenesis therapy and understand other key $\operatorname{steps}^{23}$ in suppressing tumor growth and metastasis.

For this reason, this paper will present a 3D mathematical model of development of the metastatic tumor vasculature to simulate the morphology and construction of 3D microvascular networks under the inhibitory effect of anti-angiogenic factor angiostatin excreted by the primary tumor. Microvascular density, abnormal geometric and morphological features of 3D microvasculature networks inside and outside the metastatic tumor are analyzed in the presence of angiostatin. The present 3D model may turn useful in reproducing experimental tests and can provide theoretical basis for clinical research on anti-angiogenic therapy.

\section{The 3D Mathematical Model}

The 3D mathematical model developed in this study is based on previous $2 \mathrm{D}$ tumor anti-angiogenesis mathematical models ${ }^{7,17}$ describing the formation of capillary networks inside and outside the metastatic tumor in response to anti-angiogenic factor angiostatin released by a primary tumor. The model assumes that the migration of ECs is influenced mainly by four factors ${ }^{7,24}$ : inhibitory effect of angiostatin, chemotaxis, haptotaxis and random motility of ECs, to simulate metastatic tumor angiogenesis. All parameters and equations included in the present 3D model are expressed in dimensionless form. We denote the EC density per unit area by $E$, the angiogenic stimulator concentration by $T$, the angiostatin concentration by $A$ and the fibronectin concentration by $F$. The partial differential equations governing EC motion are:

$$
\begin{gathered}
\frac{\partial E}{\partial t}=\nabla \cdot(\nabla \cdot D(r) E)-\nabla \cdot(\chi(T) E \nabla T)-\nabla \cdot(\xi(A) E \nabla A)-\nabla \cdot(\rho E \nabla F), \\
\frac{\partial T}{\partial t}=-\eta E T,
\end{gathered}
$$




$$
\begin{gathered}
\frac{\partial A}{\partial t}=-\gamma E A \\
\frac{\partial F}{\partial t}=-\omega E-\mu E F
\end{gathered}
$$

where

$$
\begin{gathered}
D(r)= \begin{cases}D_{0} & \left(r>r_{t}\right), \\
D_{0}\left(r / r_{t}\right)^{2} & \left(r \leq r_{t}\right),\end{cases} \\
\chi(T)=\chi_{0} /(1+\beta T), \\
\xi(A)=\alpha_{0} A
\end{gathered}
$$

are the EC random motility, chemotaxis and inhibitory sensitivity functions, respectively. Here, $r$ is the distance from the center of the metastatic tumor and $r_{t}$ is the radius of the metastatic tumor. Angiostatin is a potent anti-angiogenic factor that specifically inhibits EC proliferation and migration in a dose-dependent manner, i.e., the more angiostatin present in the system, the more will be the inhibition. In order to model the dose-dependent response of ECs to angiostatin, we assume the simple linear functional form for $\xi(A)$. This function reflects the increase of inhibitory sensitivity with increased angiostatin concentration. The coefficients $D_{0}, \chi_{0}$, $\beta, \alpha_{0}, \rho, \eta, \gamma, \omega$ and $\mu$ are positive constants.

The above equations hold true over the cube spatial domain that is $[0,1] \times[0,1] \times[0,1]$, which is equivalent to dimensions of $[0,2 \mathrm{~mm}] \times[0,2 \mathrm{~mm}] \times$ $[0,2 \mathrm{~mm}]$. The parent vessel is located along one edge $(x=0, y=0: 1, z=0.5)$, and the metastatic tumor is located on the opposite edge. Normal tissue surrounds the metastatic tumor, which is located at the center $(1.0,0.5,0.5)$ and has a dimensionless radius of 0.5 . Based on perfusion rate ${ }^{25}$ three regions can be recognized in the metastatic tumor: well-vascularized region $\mathrm{T} 1 \quad(0.3 \leq r<0.5)$, semi-necrotic region T2 $(0.1 \leq r<0.3)$ and necrotic region $\mathrm{T} 3 \quad(0.0 \leq r<0.1)$. Schematic modeling of $3 \mathrm{D}$ metastatic tumor angiogenesis is shown as Fig. 1.

It is assumed that the cells and, consequently, the capillary sprouts remain within the domain of the tissue. Hence, the no-flux boundary condition is

$$
\zeta \cdot(-\nabla \cdot D(r) E+\chi(T) E \nabla T+\xi(A) E \nabla A+\rho E \nabla F)=0
$$

where $\zeta$ is an appropriate outward unit normal vector.

According to previous mathematical models ${ }^{7,24}$ and considering the $3 \mathrm{D}$ model, the initial concentration profiles of the angiogenic stimulators, angiostatin and fibronectin, respectively, can be expressed as

$$
\begin{cases}T(x, y, z, 0)=e^{-\frac{(1-x)^{2}+(z-0.5)^{2}}{\varepsilon_{1}}}, & (x, y, z) \in[0,1] \times[0,1] \times[0,1], \\ A(x, y, z, 0)=s_{1} e^{-\frac{x^{2}+(z-0.5)^{2}}{\varepsilon_{2}}}, & (x, y, z) \in[0,1] \times[0,1] \times[0,1], \\ F(x, y, z, 0)=s_{2} e^{-\frac{x^{2}+(z-0.5)^{2}}{\varepsilon_{3}}}, & (x, y, z) \in[0,1] \times[0,1] \times[0,1],\end{cases}
$$




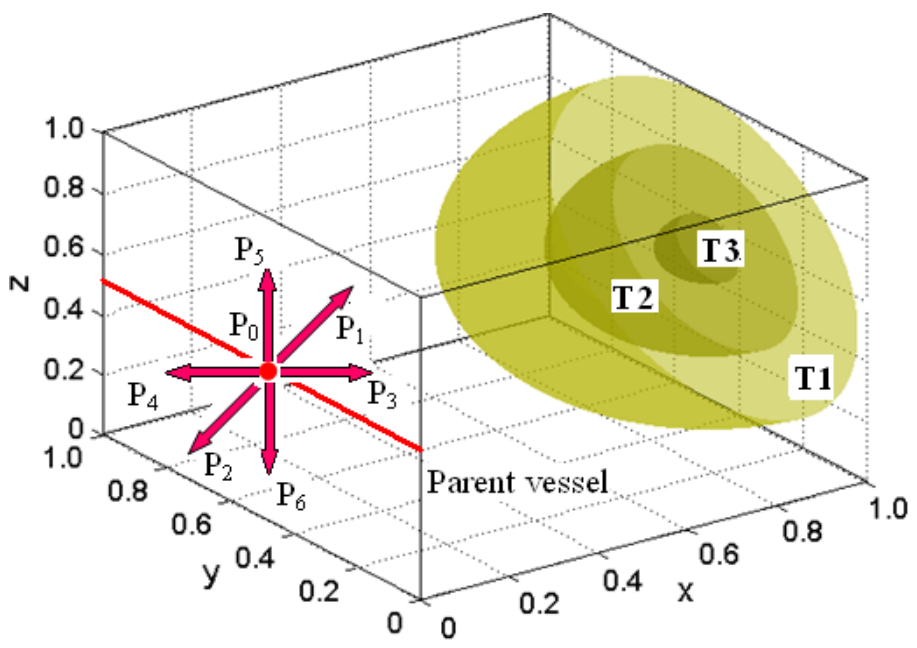

Fig. 1. Schematic modeling of metastatic tumor angiogenesis.

where $\varepsilon_{1}, \varepsilon_{2}, \varepsilon_{3}$ and $s_{2}$ are positive constants. The parameter $s_{1}=1.0$ depends on the size of the primary tumor. Also $x, y$ and $z$ are the dimensionless coordinates in the $3 \mathrm{D}$ space.

By using standard Euler central finite difference approximations, ${ }^{26}$ we obtained the $3 \mathrm{D}$ discretized equations as follows:

$$
\begin{gathered}
E_{l, m, j}^{q+1}=E_{l, m, j}^{q} P_{0}+E_{l+1, m, j}^{q} P_{1}+E_{l-1, m, j}^{q} P_{2}+E_{l, m+1, j}^{q} P_{3}+E_{l, m-1, j}^{q} P_{4} \\
+E_{l, m, j+1}^{q} P_{5}+E_{l, m, j-1}^{q} P_{6}, \\
T_{l, m, j}^{q+1}=T_{l, m, j}^{q}\left(1-k \eta E_{l, m, j}^{q}\right), \\
A_{l, m, j}^{q+1}=A_{l, m, j}^{q}\left(1-k \gamma E_{l, m, j}^{q}\right), \\
F_{l, m, j}^{q+1}=F_{l, m, j}^{q}\left(1-k \mu E_{l, m, j}^{q}\right)+k \omega E_{l, m, j}^{q},
\end{gathered}
$$

where the subscripts specify the location on the grid and the superscripts the time steps. That is, $x=l h, y=m h, z=j h$ and $t=q k$, where $l, m, j, q, h$ and $k$ are positive parameters. The migration of an individual EC located at the capillary sprout is determined by the set of coefficients $P_{0}-P_{6}$, which correspond to seven directions (see Fig. 1): stationary $\left(P_{0}\right)$ or moving into the plane $\left(P_{1}\right)$, out of the plane $\left(P_{2}\right)$, right $\left(P_{3}\right)$, left $\left(P_{4}\right)$, up $\left(P_{5}\right)$ or down $\left(P_{6}\right)$, having the below forms:

$$
\begin{aligned}
P_{0}= & 1-\frac{6 k D}{h^{2}}+\frac{k \beta \chi\left(T_{l, m, j}^{q}\right)}{4 h^{2}\left(1+\beta T_{l, m, j}^{q}\right)^{2}}\left[\left(T_{l+1, m, j}^{q}-T_{l-1, m, j}^{q}\right)^{2}+\left(T_{l, m+1, j}^{q}-T_{l, m-1, j}^{q}\right)^{2}\right. \\
& \left.+\left(T_{l, m, j+1}^{q}-T_{l, m, j-1}^{q}\right)^{2}\right]-\frac{k \chi\left(T_{l, m, j}^{q}\right)}{h^{2}\left(1+\beta T_{l, m, j}^{q}\right)}\left(T_{l+1, m, j}^{q}+T_{l-1, m, j}^{q}+T_{l, m+1, j}^{q}\right. \\
& \left.+T_{l, m-1, j}^{q}+T_{l, m, j+1}^{q}+T_{l, m, j-1}^{q}-6 T_{l, m, j}^{q}\right)-\frac{k \alpha_{0}}{4 h^{2}}\left[\left(A_{l+1, m, j}^{q}-A_{l-1, m, j}^{q}\right)^{2}\right.
\end{aligned}
$$




$$
\begin{aligned}
& \left.+\left(A_{l, m+1, j}^{q}-A_{l, m-1, j}^{q}\right)^{2}+\left(A_{l, m, j+1}^{q}-A_{l, m, j-1}^{q}\right)^{2}\right]-\frac{k \alpha_{0} A_{l, m, j}^{q}}{h^{2}}\left(A_{l+1, m, j}^{q}\right. \\
& \left.+A_{l-1, m, j}^{q}+A_{l, m+1, j}^{q}+A_{l, m-1, j}^{q}+A_{l, m, j+1}^{q}+A_{l, m, j-1}^{q}-6 A_{l, m, j}^{q}\right)-\frac{k \rho}{h^{2}}\left(F_{l+1, m, j}^{q}\right. \\
& \left.+F_{l-1, m, j}^{q}+F_{l, m+1, j}^{q}+F_{l, m-1, j}^{q}+F_{l, m, j+1}^{q}+F_{l, m, j-1}^{q}-6 F_{l, m, j}^{q}\right), \\
& P_{1}=\frac{k D}{h^{2}}-\frac{k}{4 h^{2}}\left[\frac{\chi\left(T_{l, m, j}^{q}\right)}{\left(1+\beta T_{l, m, j}^{q}\right)}\left(T_{l+1, m, j}^{q}-T_{l-1, m, j}^{q}\right)+\alpha_{0} A_{l, m, j}^{q}\left(A_{l+1, m, j}^{q}\right.\right. \\
& \left.\left.-A_{l-1, m, j}^{q}\right)+\rho\left(F_{l+1, m, j}^{q}-F_{l-1, m, j}^{q}\right)\right] \text {, } \\
& P_{2}=\frac{k D}{h^{2}}+\frac{k}{4 h^{2}}\left[\frac{\chi\left(T_{l, m, j}^{q}\right)}{\left(1+\beta T_{l, m, j}^{q}\right)}\left(T_{l+1, m, j}^{q}-T_{l-1, m, j}^{q}\right)+\alpha_{0} A_{l, m, j}^{q}\left(A_{l+1, m, j}^{q}\right.\right. \\
& \left.\left.-A_{l-1, m, j}^{q}\right)+\rho\left(F_{l+1, m, j}^{q}-F_{l-1, m, j}^{q}\right)\right] \text {, } \\
& P_{3}=\frac{k D}{h^{2}}-\frac{k}{4 h^{2}}\left[\frac{\chi\left(T_{l, m, j}^{q}\right)}{\left(1+\beta T_{l, m, j}^{q}\right)}\left(T_{l, m+1, j}^{q}-T_{l, m-1, j}^{q}\right)+\alpha_{0} A_{l, m, j}^{q}\left(A_{l, m+1, j}^{q}\right.\right. \\
& \left.\left.-A_{l, m-1, j}^{q}\right)+\rho\left(F_{l, m+1, j}^{q}-F_{l, m-1, j}^{q}\right)\right] \text {, } \\
& P_{4}=\frac{k D}{h^{2}}+\frac{k}{4 h^{2}}\left[\frac{\chi\left(T_{l, m, j}^{q}\right)}{\left(1+\beta T_{l, m, j}^{q}\right)}\left(T_{l, m+1, j}^{q}-T_{l, m-1, j}^{q}\right)+\alpha_{0} A_{l, m, j}^{q}\left(A_{l, m+1, j}^{q}\right.\right. \\
& \left.\left.-A_{l, m-1, j}^{q}\right)+\rho\left(F_{l, m+1, j}^{q}-F_{l, m-1, j}^{q}\right)\right] \text {, } \\
& P_{5}=\frac{k D}{h^{2}}-\frac{k}{4 h^{2}}\left[\frac{\chi\left(T_{l, m, j}^{q}\right)}{\left(1+\beta T_{l, m, j}^{q}\right)}\left(T_{l, m, j+1}^{q}-T_{l, m, j-1}^{q}\right)+\alpha_{0} A_{l, m, j}^{q}\left(A_{l, m, j+1}^{q}\right.\right. \\
& \left.\left.-A_{l, m, j-1}^{q}\right)+\rho\left(F_{l, m, j+1}^{q}-F_{l, m, j-1}^{q}\right)\right] \text {, } \\
& P_{6}=\frac{k D}{h^{2}}+\frac{k}{4 h^{2}}\left[\frac{\chi\left(T_{l, m, j}^{q}\right)}{\left(1+\beta T_{l, m, j}^{q}\right)}\left(T_{l, m, j+1}^{q}-T_{l, m, j-1}^{q}\right)+\alpha_{0} A_{l, m, j}^{q}\left(A_{l, m, j+1}^{q}\right.\right. \\
& \left.\left.-A_{l, m, j-1}^{q}\right)+\rho\left(F_{l, m, j+1}^{q}-F_{l, m, j-1}^{q}\right)\right] \text {. }
\end{aligned}
$$

Stochastic movement rules for the cells were directly derived from the discretized model. In particular, biased random-walk governing the motion of a single EC relies on the system of partial differential equations. Cell movement depends on the response to a chemical stimulus by considering an equation governing the probability that a cell is at a given position at a given time. This equation depends on the transition probabilities for one-step jumps to the orthogonal neighbors. These 
probabilities define the potential displacement directions for each EC located at each sprout tip.

Simulations of the discrete stochastic model are performed by calculating the coefficients $P_{0}-P_{6}$ at each time step and the probabilities define the potential displacement directions for each EC located at each sprout tip. Probability ranges are then computed by summing the coefficients to produce seven ranges $R_{0}=0-P_{0}$ and $R_{j}=\sum_{i=0}^{j-1} P_{i}-\sum_{i=0}^{j} P_{i}$, where $j=1, \ldots, 6$. We then generate a random number between 0 and 1 , and depending on the range into which this number falls, the current individual EC under consideration will remain stationary or move. The larger a particular range, the greater the probability that the corresponding coefficient will be selected. While independent simulations must be carried out as the seed of the random number generator involved in the definition of cell movements will change for each run, the step size given to the EC does not depend on variations in EC concentration because it is set equal to the mesh size, approximately the length of one or two EC. The processes of branching and anastomosis of capillary sprouts are incorporated into the discretized form of the 3D model. The finite difference approach utilized to develop the present 3D model may limit geometric complexity of parent vessel and metastatic tumor that can be analyzed. While finite element models are certainly more general in this regard, the required computational cost may increase exponentially with respect to finite difference schemes.

\section{Results and Discussion}

The simulations are performed using the finite difference method to solve partial differential equations (1)-(4) with appropriate boundary and initial conditions. The nondimensional parameter values used in the following simulations are $D_{0}=0.00035, \chi_{0}=0.38, \beta=0.6, \rho=0.34, \eta=0.1, \gamma=0.1, \omega=0.05, \mu=0.1$, $\varepsilon_{3}=0.45$ (Ref. 7) and $\alpha_{0}=0.38, \varepsilon_{1}=0.2, \varepsilon_{2}=0.6, s_{1}=1.0, s_{2}=0.75, \kappa=0.75$ (Ref. 8). More details on the definition of these parameters can be found in Refs. 7 and 8 . The same values used in the $2 \mathrm{D}$ simulations previously carried out in literature were used also for the 3D model developed here. Variations of these parameters with respect to the type of tumor, patient's gender, age and general health conditions are very important to angiogenesis in anti-angiogenic cancer therapy but could not be considered in the present study because it is very difficult to experimentally estimate changes of these parameters. Effect of biochemical signaling also should be added to the 3D model in future investigations.

The spatiotemporal dynamic growth of 3D microvascular networks inside and outside the metastatic tumor for two situations in the presence and absence of angiostatin with the same initial data and other parameters is shown in Fig. 2. At the early stage $(t=3$ days), the vessels have grown a certain distance towards the metastatic tumor, but the ECs are only quarter way through the simulation domain under the influence of angiostatin. Conversely, ECs have reached and penetrated into metastatic tumor and formed blood perfusion loops in the absence of 


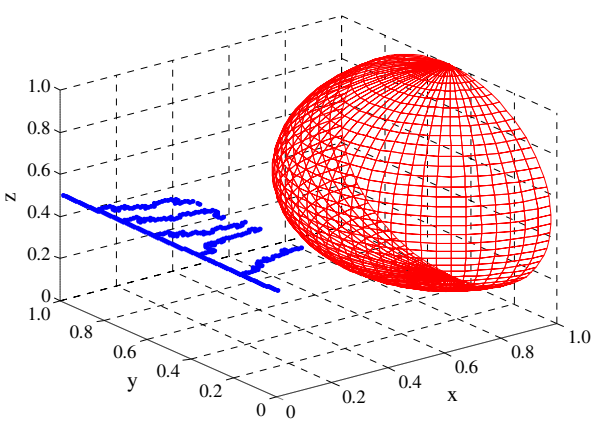

(a) $t=3$ days.

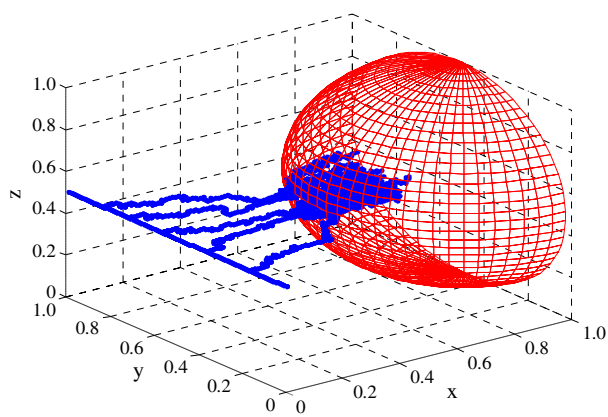

(c) $t=14$ days.

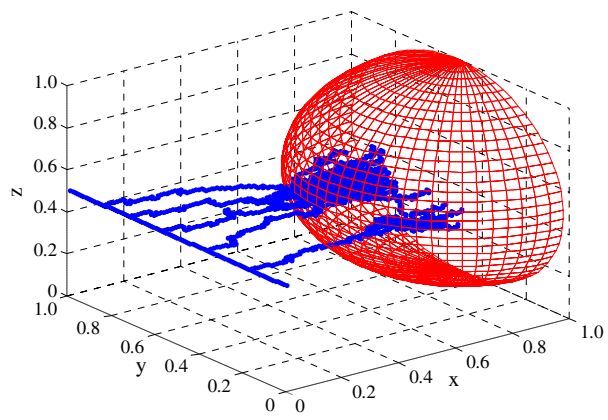

(e) $t=7$ days.

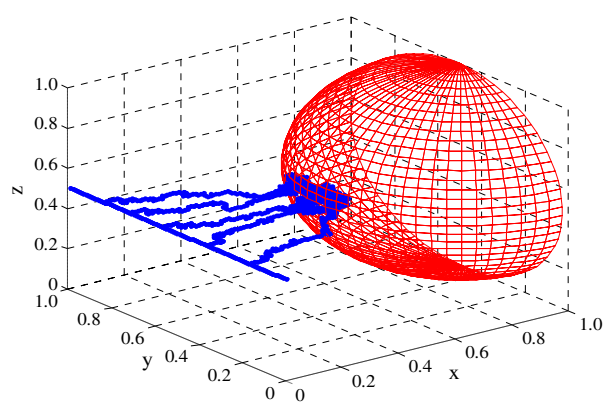

(b) $t=7$ days.

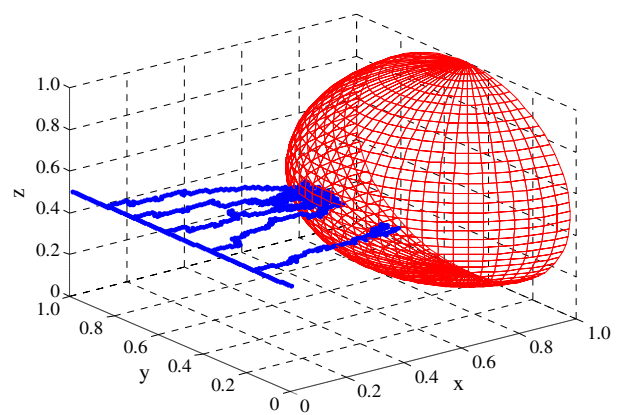

(d) $t=3$ days.

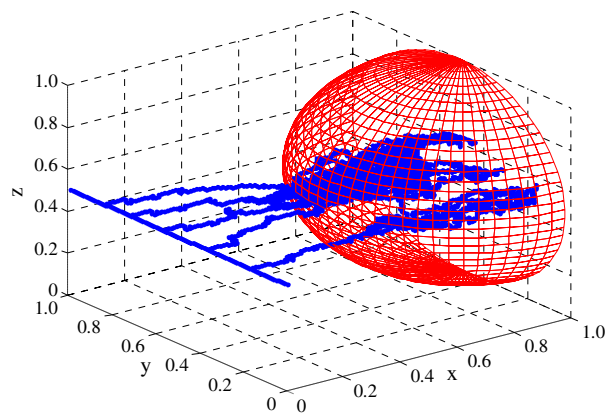

(f) $t=14$ days.

Fig. 2. The spatiotemporal dynamic growth of 3D microvascular networks inside and outside the metastatic tumor: (a)-(c) indicate growth in the presence of angiostatin; (d)-(f) indicate growth in the absence of angiostatin with the same initial data and other parameters.

angiostatin. As time progresses $(t=7$ days), the microvascular networks have some branch and anastomosis occurring and the neocapillaries gradually spread into metastatic tumor tissues in the presence of angiostatin. At the same time, the vessel density has a rapid increase forming a well-circulated region at the metastatic tumor interior in the absence of angiostatin. The extensive neovessel bed can supply the 
nutrients and oxygen for the rapid growth of metastatic tumor. Subsequently, the number of vessels speedily increases between $t=7$ days and $t=14$ days creating the dense microvascular networks which present the abnormal geometric and morphological features of 3D metastatic tumor microvasculature, such as heterogeneous density distribution, vessel tortuosity, branching and anastomosis. Comparing the plots of 3D microvascular network at $t=3,7$ and 14 days, we note that the migration of the ECs towards the metastatic tumor is slower and the microvascular density and lateral movement of the vascular sprout are less under the inhibitory effect of angiostatin. The comparison of 3D microvascular network morphology and structure shows that angiostatin excreted by the primary tumor can decrease capillary number, growth rate of microvasculature, vessel branching and anastomosis, and inhibit the extension and growth of the new capillary bed inside and outside the metastatic tumor, which are consistent with physiological observed facts. ${ }^{3,27}$

Tumor microvascular density (MVD), a measure of angiogenesis, has been shown to be a prognostic indicator that correlates with an increased risk of metastasis in various cancers such as breast, prostate and nonsmall cell lung carcinoma. In this study, microvascular density is determined in order to quantitatively evaluate the anti-angiogenesis effect of angiostatin.

Due to the randomness of simulation, the curves of the microvascular density profile are the mean value and standard deviation of the results of 20 simulations in the domain for two situations: in the presence and absence of angiostatin with the same initial data and other parameters. It can be seen from Fig. 3 that the microvascular network in the whole 3D simulation domain of metastatic tumor decreases by about $60 \%, 58 \%$ and $52 \%$, respectively, at $t=3,7$ and 14 days under the effect of anti-angiogenic factor angiostatin. The figure shows also that anti-angiogenic factor angiostatin can inhibit or block EC proliferation and migration, even at different stages of the metastatic tumor growth. Tao et al. ${ }^{28}$ have shown that when murine

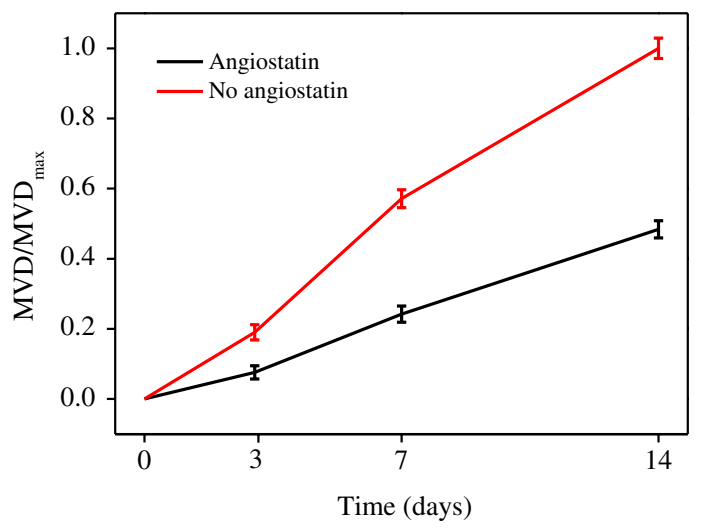

Fig. 3. Comparison of microvascular densities in the whole 3D simulation domain of metastatic tumor in the presence and absence of angiostatin at $t=3,7$ and 14 days. 
angiostatin cDNA was stably expressed in human hepatocellular carcinoma implanted in nude mice, angiogenesis as determined by microvascular density was reduced by $49 \%$ compared to untreated controls. Experimental studies where human dermal microvascular ECs were cultured showed that the number of microvascular decreased after using angiostatin. ${ }^{29}$ These results demonstrate that angiostatin has the capacity to regulate and inhibit the formation of new blood vessels inside and outside the metastatic tumor.

\section{Conclusion}

The inhibition of angiogenesis restrains nutrient supply, reducing the growth rate of the metastatic tumor and hindering migrating cell clusters from entering the vasculature, thus reducing the risk of metastasis. This paper presented a 3D mathematical model of development of metastatic tumor angiogenesis to investigate the inhibitory efficiency of anti-angiogenic factor angiostatin excreted by the primary tumor on the 3D morphology and structure of microvascular network. The simulation results demonstrate that angiostatin can decrease capillary number, growth rate, vessel branching and anastomosis, and inhibit the development and growth of microvascular networks inside and outside the metastatic tumor. Hence, angiostatin can also improve various processes related to the inhibition of oxygen and nutrient transport towards the metastatic tumor. Modeling angiogenesis related to antiangiogenic therapy is certainly a very complex task. However, the present 3D model can be considered a good theoretical basis for clinical research on anti-angiogenic cancer therapy as it provided results consistent with experimental evidence. However, future investigations will have to compare experimental data and numerical simulations over the same time period (for example, the present analysis covered only 14 days while, for example, in Ref. 28 tumor microvascular density was measured at 35 days after cancer cell injection; since no intermediate time measurements were available, the trend of variation could not be directly compared). Furthermore, more detailed spatial information on the microvascular network structure in the metastatic tumor are available from the proposed approach. However, more realistic features and complex biology factors must be incorporated in the $3 \mathrm{D}$ model, such as the anatomy and physiology of the metastatic tumor, the effects of angiostatin pharmacokinetics and the delivery of anti-angiogenic therapy drugs, the effect of patient's gender, age and general health conditions. This will contribute to improve anti-angiogenic cancer therapy.

\section{Acknowledgments}

This work is supported by the National Natural Science Foundation of China (Grant Nos. 11502146, 11572200 and 51305268), Shanghai Natural Science Foundation (No. 15ZR1429600), Shanghai City Committee of Science and 
Technology Research Program (No. 13DZ2260900) and Chenguang Plan of Shanghai (No. 13CGB09).

\section{References}

1. Folkman J, Tumor angiogenesis: Therapeutic implications, N Engl J Med 285(10):11821186, 1971.

2. Konjevic G, Stankovic S, Matrix metalloproteinases in the process of invasion and metastasis of breast cancer, Arch Oncol 14(3-4):136-140, 2006.

3. Steeg PS, Tumor metastasis: Mechanistic insights and clinical challenges, Nat Med 12(8):895-904, 2006.

4. O'Reilly MS, Holmgren L, Shing Y, Chen C, Rosenthal RA, Moses M, Lane WS, Cao Y, Sage EH, Folkman J, Angiostatin: A novel angiogenesis inhibitor that mediates the suppression of metastases by a Lewis lung carcinoma, Cell 79(2):315-328, 1994.

5. Sim BKL, O'Reilly MS, Liang H, Fortier AH, He WX, Madsen JW, Lapcevich R, Nacy $\mathrm{CA}$, A recombinant human angiostatin protein inhibits experimental primary and metastatic cancer, Cancer Res 57:1329-1334, 1997.

6. Paweletz N, Knierim M, Tumor-related angiogenesis, Crit Rev Oncol Hematol 9(3):197-242, 1989.

7. Anderson ARA, Chaplain MAJ, A gradient-driven mathematical model of antiangiogenesis, Math Comput Model 32:1141-1152, 2000.

8. Anderson ARA, Chaplain MAJ, Continuous and discrete mathematical models of tumor induced angiogenesis, Bull Math Biol 60:857-900, 1998.

9. Anderson ARA, Chaplain MAJ, McDougall S, A hybrid discrete-continuum model of tumour induced angiogenesis, in Jackson TL (ed.), Modeling Tumor Vasculature, Springer, New York, PP. 105-133, 2012.

10. Aubert M, Chaplain MAJ, McDougall SR, Devlin A, Mitchell CA, A continuous mathematical model of the developing murine retinal vasculature, Bull Math Biol 73:2430-2451, 2011.

11. Levine HA, Pamuk S, Sleeman BD, Nielsen-Hamilton M, Mathematical modeling of the capillary formation and development in tumor angiogenesis: Penetration into the stroma, Bull Math Biol 63(5):801-863, 2001.

12. McDougall SR, Anderson ARA, Chaplain MAJ, Sherratt JA, Mathematical modeling of flow through vascular network: Implications for tumor-induced angiogenesis and chemotherapy strategies, Bull Math Biol 64:673-702, 2002.

13. Stephanou A, McDougall SR, Anderson ARA, Chaplain MAJ, Mathematical modelling of flow in 2D and 3D vascular networks: Applications to anti-angiogenic and chemotherapeutic drug strategies, Math Comput Model 41:1137-1156, 2005.

14. Stephanou A, McDougall SR, Anderson AR, Chaplain MA, Mathematical modelling of the influence of blood rheological properties upon adaptative tumour-induced angiogenesis, Math Comput Model 44(1):96-123, 2006.

15. Zheng X, Wise SM, Cristini V, Nonlinear simulation of tumor necrosis, neovascularization and tissue invasion via an adaptive finite-element/level-set method, Bull Math Biol 67:211-259, 2005.

16. Jackson T, Zheng X, A cell-based model of endothelial cell migration, proliferation and maturation during corneal angiogenesis, Bull Math Biol 72:830-868, 2010.

17. Tee D, DiStefano J, Simulation of tumor-induced angiogenesis and its response to antiangiogenic drug treatment: Mode of drug delivery and clearance rate dependencies, J Cancer Res Clin Oncol 130:15-24, 2004. 
18. Maggelakis SA, The effects of tumor angiogenesis factor (TAF) and tumor inhibitor factors (TIFs) on tumor vascularization: A mathematical model, Math Comput Model 23(6):121-133, 1996.

19. Orme ME, Chaplain MA, Two-dimensional models of tumour angiogenesis and antiangiogenesis strategies, IMA J Math Appl Med Biol 14(3):189-205, 1997.

20. Plank MJ, Sleeman BD, A reinforced random walk model of tumour angiogenesis and anti-angiogenic strategies, Math Med Biol 20(2):135-181, 2003.

21. Alarcon T, Owen MR, Byrne HM, Maini PK, Multiscale modelling of tumour growth and therapy: The influence of vessel normalisation on chemotherapy, Comput Math Methods Med 7(2-3):85-119, 2006.

22. Ledzewicz U, Schättler H, Analysis of a mathematical model for tumor antiangiogenesis, Optim Control Appl Methods 29(1):41-57, 2008.

23. Ng EY, Sudharsan NM, An improved three-dimensional direct numerical modelling and thermal analysis of a female breast with tumour, Proc Inst Mech Eng H, J Eng Med 215(1):25-37, 2001.

24. Zhao G, Yan W, Chen E, Yu X, Cai W, Numerical simulation of the inhibitory effect of angiostatin on metastatic tumor angiogenesis and microenvironment, Bull Math Biol 5(2):274-287, 2013.

25. Kuszyk BS, Corl FM, Franano FN, Bluemke DA, Hofmann LV, Fortman BJ, Fishman EK, Tumor transport physiology: Implications for imaging and imaging-guided therapy, AJR Am J Roentgenol 177(4):747-753, 2001.

26. Mitchell AR, Griffiths DF, The Finite Difference Method in Partial Differential Equations, Wiley, Chichester, 1980.

27. Fukumura D, Jain RK, Tumor microvasculature and microenvironment: Targets for antiangiogenesis and normalization, Microvasc Res 74:72-84, 2007.

28. Tao KS, Dou KF, Wu XA, Expression of angiostatin cDNA in human hepatocellular carcinoma cell line SMMC-7721 and its effect on implanted carcinoma in nude mice, World J Gastroenterol 10:1421-1424, 2004.

29. Eriksson K, Magnusson P, Dixelius J, Claesson-Welsh L, Cross MJ, Angiostatin and endostatin inhibit endothelial cell migration in response to FGF and VEGF without interfering with specific intracellular signal transduction pathways, FEBS Lett 536:19-24, 2003. 